\title{
Investigaciones hidrogeológicas en la laguna Tuyajto perteneciente a la Reserva Nacional de los Flamencos (Atacama, Chile)
}

\author{
Christian Herrera Lameli(1), Javier Urrutia Meza(1), Jorge Jódar Bermúdez ${ }^{(2)}$, \\ Luis Javier Lambán Jiménez ${ }^{(2)}$, Emilio Custodio Gimena ${ }^{(3)}$ y Carolina Gamboa Palacios ${ }^{(1)}$
}

(1) Departamento de Ciencias Geológicas, Universidad Católica del Norte, Antofagasta, Chile. cherrera@ucn.cl; jum002@ucn.cl; cgp010@alumnos.ucn.cl

(2) Instituto Geológico y Minero de España, Unidad de Zaragoza, C/ Manuel Lasala, 44, 50006 Zaragoza, España. javier.lamban@igme.es; j.jodar@igme.es

(3) Real Academia de Ciencias. Grupo de Hidrología Subterránea, Departamento de Ingeniería Civil y Ambiental, Universidad Politécnica de Cataluña. C/ Jordi Girona, 1 - 3 UPC Campus Nord, Edificio D2, 08034 Barcelona, España. emilio.custodio@upc.edu

\section{RESUMEN}

La Laguna Tuyajto es un lago salino ubicado en la zona meridional de la reserva nacional de los flamencos (Atacama, Chile), en el Altiplano Andino del Norte de Chile. Esta laguna se encuentra a los pies del volcán Tuyajto. Está alimentada por una serie de manantiales que se encuentran en la zona Norte y Este de la laguna, que descargan agua procedente de un acuífero en materiales volcánicos recientes. El área se ubica en una

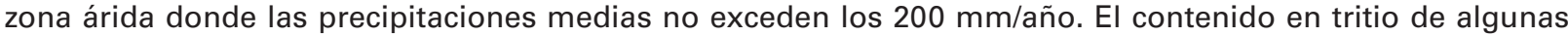
muestras de agua indica la presencia en el acuífero de aguas de recarga con tiempos de tránsito cortos. Esta recarga procede de la infiltración del agua de fusión de nieve y en menor medida del agua procedente de eventos cortos pero intensos de precipitación en el verano austral. Los gradientes verticales de precipitación y su contenido isotópico $\left(\delta^{18} \mathrm{O}\right.$ y $\left.\delta^{2} \mathrm{H}\right)$ muestran que la zona de recarga de los manantiales ubicados al Norte de la laguna se encuentra en las laderas del volcán Tuyajto por encima de los 4900 m s.n.m., mientras que en los manantiales ubicados al Este de la laguna la recarga se produce a altitudes entre 4400 y 4700 m s.n.m. y procede de las cuencas aledañas de Pampa Colorada y Pampa Las Tecas. El agua de estos manantiales puede contener tritio medible. La composición química de las aguas subterráneas es el resultado de los procesos de evaporación de aguas meteóricas, interacción agua-roca a alta temperatura y la disolución de depósitos de antiguos salares enterrados. El nivel de las aguas subterráneas es somero, debido a la presencia de una capa ignimbrítica de baja permeabilidad que inhibe la formación de celdas convectivas de flujo de agua subterránea por efecto de la alta densidad de la salmuera. A nivel local, el funcionamiento de la laguna deTuyajto es flujo de tránsito (flow-through) respecto al flujo regional del acuífero. La permanencia de los manantiales es esencial para la existencia de la lámina de salmuera y el mantenimiento de las condiciones ecológicas en cuanto a la avifauna.

Palabras clave: Laguna Tuyajto, Agua subterránea, Volcanes, Zonas áridas, Parque Nacional.

\section{Hydrogeological research in the Tuyajto Lake at the Flamingo National Reserve (Atacama, Chile)}

\section{ABSTRACT}

The Tuyajto Lake is a saline lake located in the Andean Altiplano of northern Chile, at the foot of the volcano of the same name. It is fed by springs located on its eastern and northern boundaries. These springs discharge groundwater from a volcanic aquifer. Arid conditions dominate in the area, with average precipitation less than $200 \mathrm{~mm} /$ year. The tritium content in some groundwater samples shows the contribution of modern recharge to the total groundwater flow. Recharge occurs by infiltration of snowmelt in the austral winter months and to a lesser extent by short but intense precipitation events during the summer. The vertical gradients of rainfall isotopic content $\left(\delta^{18} \mathrm{O}, \delta^{2} \mathrm{H}\right)$ and precipitation points to the recharge zone of the springs being 
located at the northern area of the lake, above 4,900 $\mathrm{m}$ a.s.l. along the slopes of the Tuyajto volcano, whereas recharge to the springs discharging on the eastern area of the lake originates in the adjacent basins of Pampa Colorada and Pampa Las Tecas at altitudes between 4,400 and 4,700 $\mathrm{m}$ a.s.l. The water of these springs may contain measurable tritium. The chemical composition of groundwater is the result of meteoric water evaporation processes, high temperature water-rock interaction and dissolution of buried old salt flat deposits. The groundwater flow is shallow, due to the presence of a regional low permeability ignimbrite formation that precludes the formation of deep convective groundwater flow cells due to the high density of brines. At a local scale, the Tuyajto lake behaves as a flow-through system with respect to the regional groundwater flow. The persistence of the springs is essential for the existence of the brine sheet and maintaining the ecological conditions for the waterfowl.

Keywords: Tuyajt lake, groundwater flow, volcanic formations, arid area, National Park.

\section{ABRIDGED ENGLISH VERSION}

\section{Introduction}

The Laguna Tuyajto basin is located in the Andean Altiplano of northern Chile, which corresponds to an active volcanic environment with altitudes that in most cases exceeds $4,000 \mathrm{~m}$ a.s.l. (Fig. 1). This area has a large number of endorheic basins containing saline and salt lakes and lagoons in their central parts (Chong, 1988; Risacher et al., 2003) and valuable water resources. Recently, the amount of research in this area has increased in order to use local groundwater resources to satisfy the high demand caused from the development of urban centres and agricultural, tourist and mining activities. In 2003, a multidisciplinary study started in the Laguna Tuyajto basin by water management consultants (WMC, 2006), which consisted of evaluating the water balance through hydrological, hydrogeological, hydrochemical and environmental isotope techniques.

The aquifers located in the Laguna Tuyajto basin and in its surroundings, are protected by the Chilean government, because they allow the existence of valuable but fragile groundwater dependent wetlands, locally known as "vegas" and "bofedales". In addition, these ecosystems are very important to populations in the Altiplano, because they have used these water resources for a very long time for their survival. Despite the importance of these systems, the knowledge about their functioning and the role of the different processes that condition the water balance are scarce. So, the main objective of this study is to contribute to the knowledge of surface and groundwater interaction in an endorheic basin within the active volcanic environment of the Central Andes, taking as a case study the Laguna Tuyajto basin. A conceptual model that characterizes the functioning of the hydrological system is presented, explaining the origin of dissolved solutes in groundwater and the possible lateral water transfer between neighbouring basins. This conceptual model can be extrapolated to other similar basins of the Andean Altiplano (e.g. the Salar de Pujsa), which have been declared RAMSAR sites according to their importance and singularity.

\section{The Study Area}

The Laguna Tuyajto basin is an endorheic basin located in the Andean Altiplano of the Antofagasta Region (Fig. 1a). It is limited to the west by the Salar de Aguas Calientes 3 and to the east by the Pampa Colorada and Pampa Las Tecas basins (Fig. 1b). The climate is arid, with an average precipitation that between 150 and 200 $\mathrm{mm} /$ year (DGA, 2009), an average temperature of $1{ }^{\circ} \mathrm{C}$ and an average potential evaporation of 1,500 $\mathrm{mm} /$ year (Risacher et al., 1999).

From a geological point of view, the study area is dominated by the volcanic activity developed from the Miocene to the Present. Volcanoes are well preserved and overlay a regional ignimbritic plateau formed in the Upper Miocene, which can reach $100 \mathrm{~m}$ in thickness (Mardones, 1977; Stern et al., 2007). This ignimbrite unit is considered to be associated with a large caldera in the central Andes, called La Pacana (Backer, 1981; Ramirez and Gardeweg, 1982; Lindsaya et al., 2001). In addition, four types of lava can be distinguished: dacites, hornblende and / or biotite bearing andesites, pyroxene bearing andesites, and basalts. The ignimbritic formations associated to recent volcanism, generated after the large plateau mentioned above, are the Guimquitin (Upper Miocene) and Chacana ignimbrites (Upper Pliocene), recognized both to the east of the study area (in Pampa Colorada and Pampa Las Tecas), and the Tuyajto ignimbrite (Pleistocene-Holocene) which outcrops in the western part of the Laguna Tuyajto (Fig. 2).

The hydraulic transmissivity values range between 3 and 1,000 $\mathrm{m}^{2} /$ day. The highest values correspond to wells in the central part of Pampa Colorada and Pampa Las Tecas basins and are attributed to the existence 
Christian Herrera Lameli, et al., 2019. Investigaciones hidrogeológicas en la laguna... Boletín Geológico y Minero, 130 (4): $789-806$

of unconsolidated alluvial fans with volcanic intercalations. The ignimbrite plateau seems to play the role of an impermeable base formation for the Laguna Tuyajto aquifer (Montgomery et al., 2003).

\section{Materials and methods}

Spring water that discharges in the eastern and northern part of Laguna Tuyajto, together with two springs in the Salar de Aguas Calientes 3, were sampled. Temperature, $\mathrm{pH}$ and electric conductivity were measured in the field for each spring. The anions were analyzed by ion chromatography $(\mathrm{Cl}, \mathrm{Br})$, absorption spectrometry $\left(\mathrm{SO}_{4}, \mathrm{NO}_{3}\right)$ and titration $\left(\mathrm{HCO}_{3}, \mathrm{H}_{2} \mathrm{CO}_{3}\right)$, whereas the cations were measured by atomic absorption spectrometry. These observations were complemented by 31 chemical and isotopic analyses previously performed by WMC (2006), which includes water samples from wells in the Laguna Tuyajto, Pampa Colorada and Pampa Las Tecas basins. The ionic balance error of chemical analyses is less than $10 \%$. All $\delta^{18} \mathrm{O}$ and $\delta^{2} \mathrm{H}$ analysis were carried out at the Chilean Nuclear Energy Commission, with measurement errors of $\pm 0.05 \%$ for $\delta^{18} \mathrm{O}$ and \pm $1 \%$ for $\delta^{2} \mathrm{H}$. Values are given relative to the $\mathrm{V}$-SMOV standard.

Accumulated rainfall was sampled in three locations for the analysis of major ions and the isotopic composition of $\delta^{18} \mathrm{O}$ and $\delta^{2} \mathrm{H}$ : Salar de Aguas Calientes 3 (3900 $\mathrm{m}$ a.s.l.), Laguna Tuyajto (4040 $\mathrm{m}$ a.s. I.) and Pampa Colorada (4426 $m$ a.s.l.). A total of 10 rain samples were obtained, corresponding to January, March, May and December of 2004 and January of 2005. Additionally, snow samples were taken along a transect that runs along the slope of the Tuyajto volcano, between 3,850 and 4,500 m a.s.l., in which only the isotopic content was analyzed. The snow samples were obtained in July (Laguna Tuyajto) and August (Laguna Tuyajto, Salar de Aguas Calientes 3, Pampa Colorada and Pampa Las Tecas) of 2004.

The discharge flow of springs was measured monthly during one year at Stations A and B (Fig. 1). The discharge flow of the M-3 spring was measured on only four occasions due to its sporadic discharge. A continuous record of the Laguna Tuyajto water level was obtained by installing a pressure sensor in the deepest part. To determine evaporation, a type A evaporation tank was installed at 4,100 $\mathrm{m}$ a.s.l., in the north of the study area.

\section{Results and discussion}

The greatest water contribution to Laguna Tuyajto comes from the springs located on its northern and eastern side. The total discharge of the springs fluctuates between 74 and $245 \mathrm{~L} / \mathrm{s}$, with an estimated total contribution of $4.4 \mathrm{hm}^{3}$ in 2004 (Table 1). The piezometric map (Fig. 5) shows a hydraulic connection between Laguna Tuyajto, Pampa Las Tecas and Pampa Colorada basins, with a groundwater flow direction from east to west. The small piezometric gradients near LagunaTuyajto, in the central areas of Pampa Las Tecas and Pampa Colorada, point to high transmissivity values, possibly associated with permeable sedimentary deposits, such as gravels of alluvial fans.

From a hydrochemical point of view, local groundwater is of the sodium chloride type, with a slight excess of sulphate with respect to the marine ratio in some samples. The waters of the springs located on the eastern side of Laguna Tuyajto are also of the sodium chloride type and have the same chemical fingerprint as the groundwater in Pampa Colorada and Pampa Las Tecas basins (Fig. 6). The springs located in the northern area of Laguna Tuyajto and the Salar de Aguas Calientes 3 are of sodium chloride-sulfate type. There is a slight excess of chloride relative to sodium, especially for waters with relatively high salinity, which tends to disappear in samples with very high salinity (Fig. 7). The values of the $\mathrm{rCl} / \mathrm{rBr}$ ( $r$ means meq/L) ratio in Pampa Las Tecas and Pampa Colorada vary from 2000 to 10,000, which is characteristic of halite dissolution (Fig. 8).

The values of $\mathrm{rSO}_{4} / \mathrm{rCl}$ in rain samples vary from 0.26 to 0.28 , whilst in the springs it ranges from 0.16 to 0.54 (Fig. 9). The groundwater presents values from 0.01 to 0.45 . Waters with lower values of $r \mathrm{SO}_{4} / \mathrm{rCl}$ have a chemical composition close to rain samples, whilest some high values found in dilute water can be explained by the weathering of the volcanic rocks containing sulphides. Other high values of $\mathrm{rSO}_{4} / \mathrm{rCl}$, which are not found in dilute water, may be the product of the atmospheric diffusion of $\mathrm{SO}_{2}$ from native sulphur oxidation in a solphataric alteration zone present at the top of the Tuyajto volcano. The incorporation of $\mathrm{HCl}$ contributed by endogenous gases cannot be ruled out.

The isotopic composition $\left(\delta^{18} \mathrm{O}\right)$ of snow ranges from $-6.52 \%$ to $-10.93 \%$, with a $\delta^{18} \mathrm{O}$ isotopic vertical gradient of $-2 \%$ o $\mathrm{km}$. The $\delta^{18} \mathrm{O}$ of rainwater ranges between $-1.84 \%$ and $-10.31 \%$ (Fig. 10). The deuterium excess is $15.7 \%$. The isotopic composition of $M-1, M-3, M-4$ and $M-5$ springs are very close to local meteoric water, which indicates that the recharge of these springs comes mainly from snowmelt on the slopes of the Tuyajto volcano. The isotopic composition of water from M-2, M-7 and M-6 springs points towards an evaporation process. The groundwater samples of Pampa Colorada, Pampa Las Tecas and the M-8 spring have their isotopic compositions enriched in $\delta^{18} \mathrm{O}$ with respect to meteoric water. Considering that this is an active volcanic 
zone, an explanation could be that the original meteoric water recharging these waters has been affected by deep high temperature isotopic exchange. Preferential recharge altitudes between 4,100 and 4,600 m a.s.l. are obtained if the $\delta^{2} \mathrm{H}$ isotopic composition is considered. For a recharge area between 400 and $600 \mathrm{~km}^{2}$ and average spring discharge of $3 \mathrm{hm}^{3} / \mathrm{year}$, the average recharge can be roughly estimated between 5 and 7.5 $\mathrm{mm} / \mathrm{year}$, which corresponds to 2 to $4 \%$ of average precipitation. This recharge is produced mainly by snowmelt of snow precipitated during the austral winter (Ammann et al., 2001), due to cold frontal systems from the Pacific Ocean (Vuille and Ammann, 1997). The isotopic composition of $\delta^{18} \mathrm{O}$ and $\delta^{2} \mathrm{H}$ in precipitation and groundwater samples shows how snowmelt along the Tuyajto volcano plays an important role in the aquifer recharge. This recharge is added to that produced by rainfall along the slope. As a result, the spring water that discharges at low levels is less saline and isotopically lighter than local recharge water (Custodio and Jódar, 2016). At local level, the Laguna Tuyajto functioning is of the flow-through type with respect to the regional groundwater flow.

\section{Conclusions}

The chemical composition of springs and wells samples of Laguna Tuyajto, Pampa Colorada and Pampa las Tecas basins are controlled by the possible dissolution of buried old evaporite minerals deposited by the evaporation of meteoric waters, and partly by high temperature water-rock interaction. Dissolution of halite and possibly of sulphates can be associated with ancient salt deposits covered by volcanic flows. The isotopic composition of spring water samples located at the northern and eastern part of Laguna Tuyajto indicates that recharge water comes basically from snowmelt accumulated at high altitude on the Tuyajto volcano. This snow comes mainly from precipitation events generated by the cold fronts from the Pacific Ocean during the winter.

The spring water located on the eastern side of Laguna Tuyajto is the result of the mixture of recharge water derived from precipitation with groundwater coming from Pampa Las Tecas and Pampa Colorada basins as lateral groundwater transfer.

Locally, the Laguna Tuyajto is of flow-through type with respect to the regional aquifer. Their existence and dynamics depends essentially on spring flow contributions and partly from lateral groundwater transferred from Pampa Las Tecas and Pampa Colorada. The preservation of the ecological values and avifauna in Laguna Tuyajto is therefore closely linked to land management and the possible exploitation of local groundwater.

\section{Introducción}

En el Altiplano Andino del Norte de Chile se concentran un gran número de cuencas endorreicas en cuyas depresiones se localizan lagos salinos, lagunas y salares (Chong, 1988; Risacher et al, 2003). La mayoría de estas cuencas endorreicas están situadas en un ambiente volcánico activo, a altitudes que en la mayoría de los casos exceden los 4000 m s.n.m. (Fig. 1). Recientemente se ha incrementado el número de investigaciones en esta zona realizadas con el objetivo de aprovechar los recursos de agua disponibles para abastecer el desarrollo de centros urbanos y actividades agrícolas y mineras. La actividad minera es importante para el desarrollo del país y ha dado lugar a un número elevado de informes ambientales con el fin de conseguir permisos de explotación de agua subterránea para resolver sus necesidades. En el año 2003 se inició un estudio multidisciplinario en la cuenca de la Laguna Tuyajto, llevada a cabo por Water Management Consultants (WMC, 2006), para evaluar el balance hídrico de la Laguna Tuyajto mediante una aproximación multidisciplinar, la cual incluía técnicas hidrológicas, hidrogeológicas, hidroquímicas e isotópicas ambientales.

Los acuíferos de la Laguna Tuyajto y sus alrededores se encuentran protegidos, debido a que su funcionamiento natural permite la existencia de humedales alimentados por la descarga de aguas subterráneas. Estos humedales, conocidos localmente como vegas y bofedales sostienen una gran diversidad biológica. Además, estos ecosistemas tienen una gran importancia social, cultural y económica, ya que desde tiempos ancestrales estos humedales proporcionan un recurso hídrico vital a las poblaciones originarias del Altiplano. A pesar de su importancia, poco se sabe del funcionamiento y del papel que juegan los diferentes procesos que condicionan el balance hídrico de estos sistemas hidrológicos.

El objetivo principal del presente trabajo es ser un aporte en la comprensión de la interacción entre aguas superficiales y subterráneas en una cuenca endorreica dentro del contexto volcánico activo de los Andes Centrales, tomando como caso de estudio la Laguna Tuyajto. El modelo conceptual aquí presentado caracteriza el funcionamiento del sistema hidroló- 
gico compuesto por el acuífero y la laguna y establece un balance hidrológico que permite explicar tanto el origen de los solutos disueltos en el agua subterránea como las posibles transferencias laterales de agua entre cuencas hidrogeológicas vecinas. Conocer el funcionamiento del sistema hídrico es esencial para mantener los valores ecológicos asociados a la laguna salada y la avifauna que soporta. El modelo conceptual puede extrapolarse a otras cuencas similares del Altiplano Andino (por ejemplo, el Salar de Pujsa), que por su importancia se han declarado sitio RAMSAR. Estas cuencas tienen, presumiblemente un comportamiento hidrogeológico similar debido a la configuración del relieve, marcada por las erupciones volcánicas desde los $10 \mathrm{Ma}$ hasta el presente, y a la variabilidad climática, caracterizada por lluvias durante el verano austral debido al llamado y característico "invierno altiplánico" y por nieve durante el invierno por frentes fríos del Océano Pacífico, aunque existen diferencias, principalmente en la geología.

\section{Marco geográfico y climático}

La cuenca de la Laguna Tuyajto se ubica en el Altiplano Andino de la Región de Antofagasta (Fig. 1a). Es una cuenca endorreica que está limitada al Oeste por el Salar de Aguas Calientes 3 y al Este por las cuencas Pampa Colorada y Pampa Las Tecas. Una característica importante de esta zona es su clima árido, con una precipitación media entre 150 y 200 $\mathrm{mm} / \mathrm{año} \mathrm{(DGA,} \mathrm{2009).} \mathrm{El} \mathrm{gradiente} \mathrm{vertical} \mathrm{de} \mathrm{precipi-}$ tación en la zona de estudio es de $6 \mathrm{~mm} / \mathrm{año} / 100 \mathrm{~m}$. En la Laguna Tuyajto la cota es de 4010 m s.n.m., la temperatura media es de $1^{\circ} \mathrm{C}$ y la evaporación potencial media es de 1500 mm/año (Risacher et al., 1999).
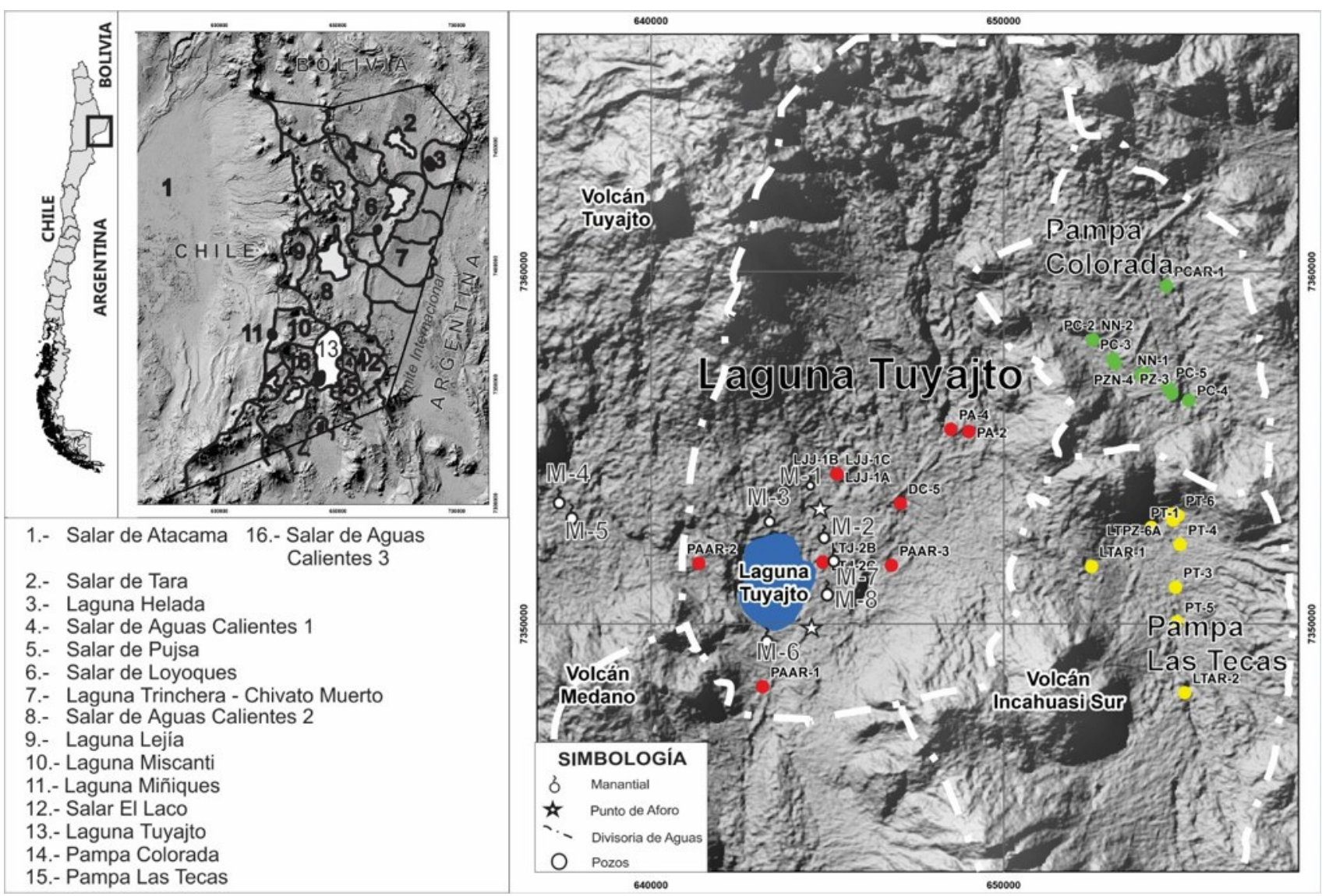

Figura 1. Mapa de ubicación del área de estudio. Situación de las cuencas significativas con salares o lagunas en condiciones áridas y semiáridas de los Andes Centrales. Los puntos rojos, amarillos y verdes indican pozos ubicados en las cuencas hidrográficas de Tuyajto, Pampa las Tecas y Pampa Colorada, respectivamente.

Figure 1. Location map of the study area. Location of the significant basins with salt flats or lakes in arid and arid-semi-arid conditions of Central Andes. Red, yellow and green points indicate wells located respectively in the Tuyajto, Pampa las Tecas and Pampa Colorada watersheds. 
La cuenca de la Laguna Tuyajto (número 13 de la Fig. 1) abarca un área total aproximada de $245 \mathrm{~km}^{2}$. En el centro de la cuenca se encuentra la Laguna Tuyajto, que recibe aportes de la descarga de cuatro manantiales principales permanentes (Tabla 1) y otros menores esporádicos. Todos ellos están ubicados en los sectores Norte y Este de la laguna.

La laguna es un cuerpo de agua libre salina con una profundidad media de $35 \mathrm{~cm}$. La superficie de agua experimenta significativas variaciones estacionales; en los meses del otoño e invierno australes alcanza un máximo de $2,7 \mathrm{~km}^{2}$, mientras que en los meses de verano se reduce a $1,7 \mathrm{~km}^{2}$. Esta importante variación se debe a tres factores: a) la suave pendiente topográfica desde la orilla hacia el centro de la laguna, lo que implica grandes variaciones de superficie con pequeños cambios del nivel, b) las variaciones estacionales de la descarga de los manantiales y c) la variación estacional de la evaporación, que durante los meses de invierno puede reducirse considerablemente cuando la superficie de la laguna está congelada.

\section{Marco geológico e hidrogeológico}

La geología del área de estudio está dominada por la actividad volcánica desarrollada desde el Mioceno al Presente (Fig. 2). La mayoría de los volcanes se encuentran bien conservados sobre una meseta formada por ignimbritas de extensión regional, que puede alcanzar los $100 \mathrm{~m}$ de espesor (Mardones, 1977; Stern et al., 2007). Esta ignimbrita se formó en el Mioceno Superior y se considera que está asociada a una gran caldera en los Andes Centrales, llamada La Pacana (Backer, 1981; Ramírez y Gardeweg, 1982; Lindsaya et al., 2001) (Fig. 2). Petrográficamente se distinguen cuatro tipos principales de lava: dacitas, andesitas con alto contenido en hornblenda y/o biotita, andesitas con alto contenido en piroxeno y basaltos. Los estratovolcanes más importantes son el Incahuasi y el Médano Sur, ubicados al Sur del área de estudio y del Mioceno Superior y el volcán Tuyajto, del Plioceno-Pleistoceno, que se encuentra justo al Norte de la Laguna Tuyajto. Las formaciones ignimbríticas asociadas al volcanismo reciente, posteriores a la gran formación antes mencionada, son la Ignimbrita Guaiquitina (Mioceno Superior) y la Ignimbrita Chacana (Plioceno Superior), reconocidas al Este del área de estudio en las cuencas Pampa Colorada y Pampa Las Tecas, y la Ignimbrita Tuyajto (Pleistoceno-Holoceno) correspondiente a una de las unidades más jóvenes de la zona (Ramírez y Gardeweg, 1982) y que aflora en la parte occidental de la Laguna Tuyajto.

La erosión que afecta a los diferentes volcanes ha producido una gran cantidad de depósitos de aluviones dispuestos en abanicos aluviales que bordean el límite Este de la Laguna Tuyajto. Estos depósitos indican que la Laguna Tuyajto fue un lago profundo hace 8000 años, alcanzando $25 \mathrm{~m}$ de profundidad (Geyh et al., 1999).

Como resultado de la interpretación de los ensayos de bombeo realizados durante diversas campañas de reconocimiento de aguas subterráneas en el Altiplano de la región de Antofagasta (WMC, 2006) se han obtenido valores de transmisividad que varían entre 3 y $1000 \mathrm{~m}^{2} /$ día. Los valores más elevados se corresponden a pozos de la parte central de las cuencas de Pampa Colorada y Pampa Las Tecas y se atribuyen a la existencia de abanicos aluviales con materiales no consolidados y con intercalaciones volcánicas. La base ignimbrítica regional parece funcionar como zócalo impermeable para el acuífero de la Laguna Tuyajto (Montgomery et al., 2003). En los reconocimientos geológicos de campo se ha compro-

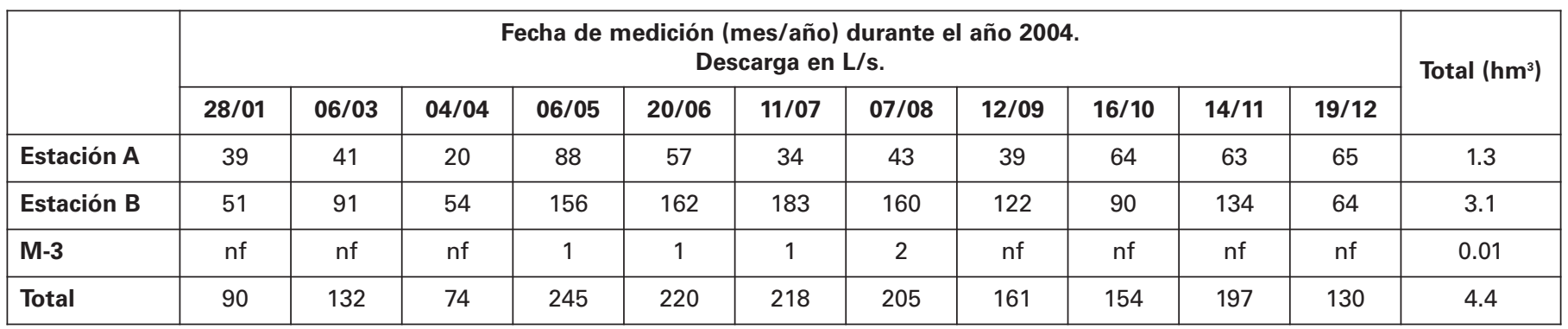

Tabla 1. Caudales de los manantiales de Laguna Tuyajto. La estación A mide el manantial M-1 y la estación B mide el flujo conjunto de los manantiales M-2, M-6-M-7 y M-8. nf = sin flujo.

Table 1. Flow rates of Laguna Tuyajto springs. Station A measures spring $M-1$ and station $B$ measures the joint flow of springs $M-2, M-6-M-$ 7 and $M-8 . n f=$ no flow. 


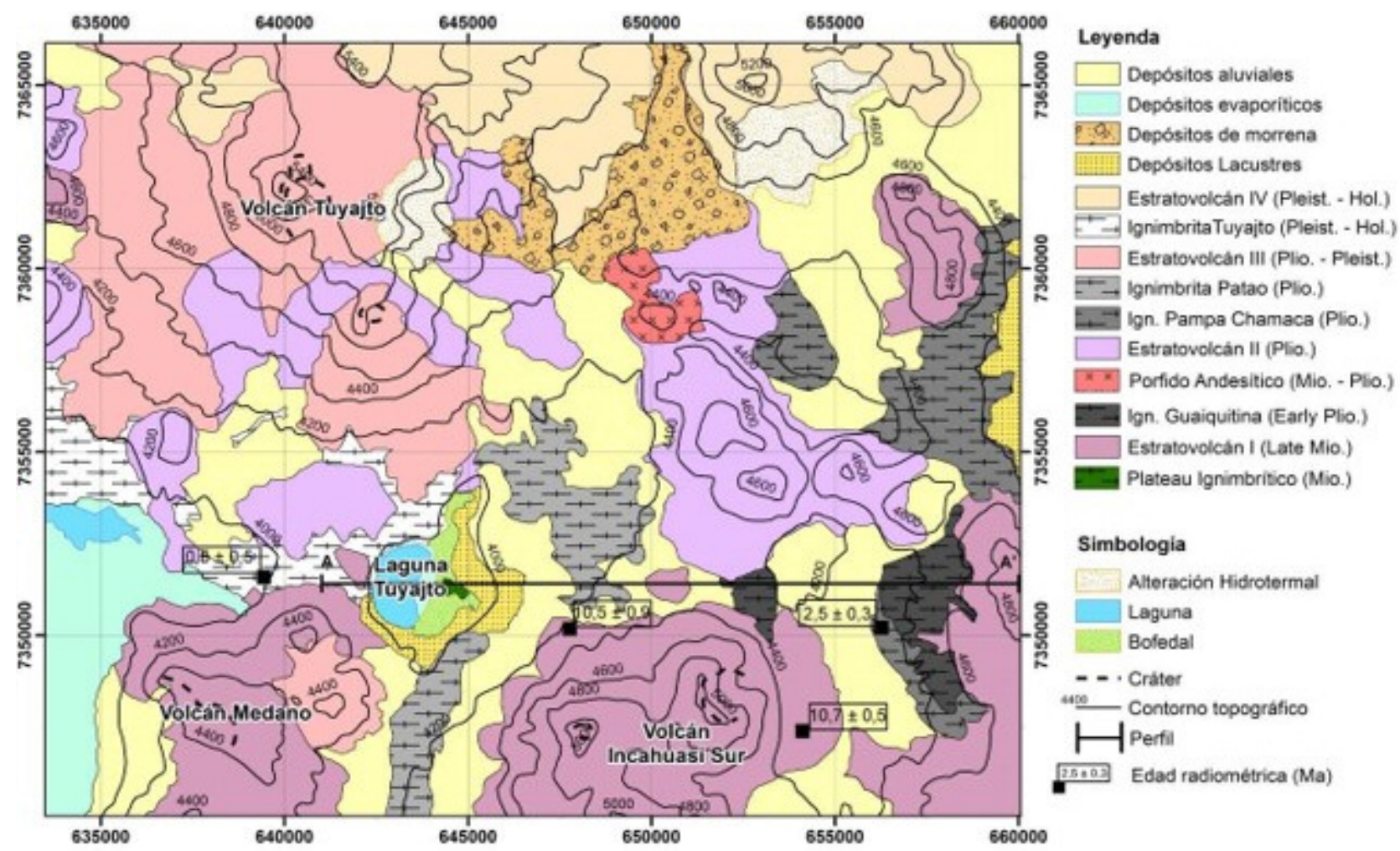

Figura 2. Mapa geológico del área de estudio. El perfil presentado en la Figura 3 se indica con las letras A-A'.

Figure 2. Geological map of the study area. The profile shown in Figure 3 is indicated by the letters A-A.'

bado que la presencia de esta ignimbrita basal genera la aparición de los manantiales anteriormente descritos (Fig. 3).

\section{Métodos y materiales}

Para la caracterización hidrogeoquímica e isotópica del sistema hidrológico de Tuyajto se ha muestreado el agua de la laguna, de los manantiales que descargan en el límite Este y Norte de la Laguna y de los dos manantiales que hay en el Salar de Aguas Calientes 3. La temperatura $(T), \mathrm{pH}$ y conductividad eléctrica (CE) de estas muestras fueron medidas in situ. Los aniones se han analizado mediante cromatografía iónica $(\mathrm{Cl}, \mathrm{Br})$, espectrometría de absorción $\left(\mathrm{SO}_{4}, \mathrm{NO}_{3}\right)$ y valoración de la alcalinidad del agua $\left(\mathrm{HCO}_{3}, \mathrm{H}_{2} \mathrm{CO}_{3}\right)$, y los cationes por espectrometría de absorción atómica.

Las muestras de agua de los manantiales de la cuenca de la Laguna Tuyajto (M-1, M-2, M-7, M-8 y M6) fueron obtenidas en diciembre de 2003 , mientras que la de los manantiales del Salar de Aguas Calientes 3 (M-4 y M-5) lo fueron en marzo de 2004.
Estas observaciones se han complementado con 31 análisis químicos e isotópicos realizados previamente por WMC (2006), los cuales incluyen muestras de agua de pozos en las cuencas Tuyajto, Pampa Colorada y Pampa Las Tecas obtenidas entre diciembre del 2004 y marzo del 2005. El error en el balance iónico de los análisis químicos es inferior a $10 \%$. Todos los análisis de $\delta^{18} \mathrm{O}$ y $\delta^{2} \mathrm{H}$ se han realizado en los laboratorios de la Comisión Chilena de Energía Nuclear, con errores de medida de $\pm 0.05 \%$ o para el $\delta^{18} \mathrm{O}$ y de $\pm 1 \%$ o para el $\delta^{2} \mathrm{H}$. Los valores se dan relativos al estándar V-SMOW.

La lluvia se ha muestreado en tres emplazamientos diferentes: Salar de Aguas Calientes $313900 \mathrm{~m}$ s.n.m.), Laguna Tuyajto (4040 m s.n.m.) y Pampa Colorada (4426 m s.n.m.). Las muestras corresponden a lluvia acumulada, en toma-muestras con una capa de vaselina para evitar la evaporación del agua acumulada a lo largo del periodo de muestreo. Las muestras de lluvia corresponden a los meses de enero, marzo y mayo del 2004 y diciembre-enero de 2005. También se recuperaron muestras de nieve en julio (Laguna Tuyajto) y agosto (Lagua Tuyajto, Salar de Aguas Calientes 3, Pampa Colorada y Pampa Las 


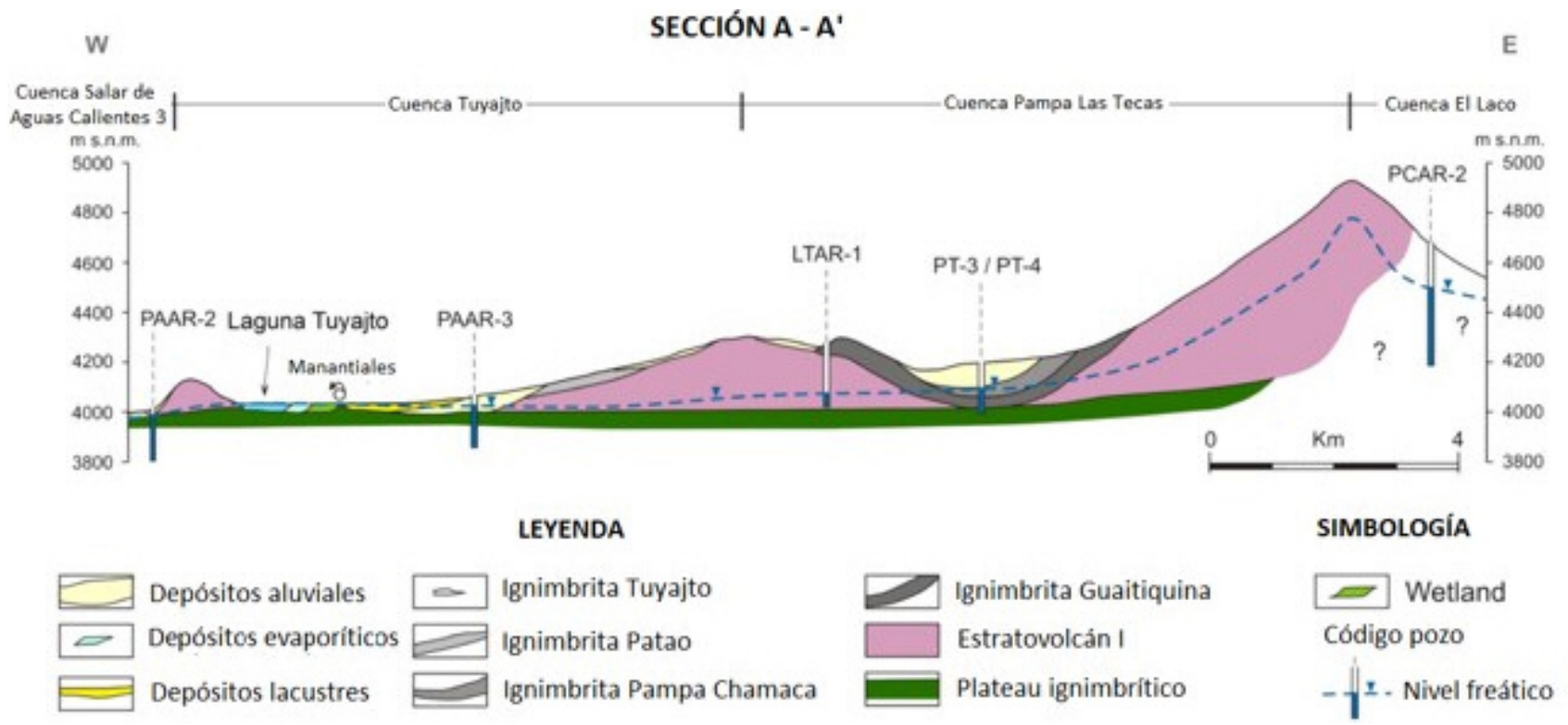

Figura 3. Sección geológica esquemática del área de estudio. Información obtenida mediante campañas de terreno, cartas geológicas regionales y descripción de pozos del estudio WMC (2006).

Figure 3. Schematic geological section of the study area. The information was obtained by means of field campaigns, regional geological maps, and well descriptions from the WMC (2006) study.

Tecas) de 2004. Se obtuvieron un total de 10 muestras de lluvia, en las que se han analizaron los iones mayoritarios y el contenido isotópico de $\delta^{18} \mathrm{O}$ y $\delta^{2} \mathrm{H}$. Adicionalmente se han tomado tres muestras de nieve a lo largo de un transecto vertical que discurre a lo largo de la ladera del volcán Tuyajto, a altitudes de 4040, 4356 y 4412 m s.n.m., en las que solo se ha podido analizar el contenido isotópico.

El caudal de descarga de los manantiales se ha medido mensualmente durante un año en las estaciones $A$, aguas abajo del manantial $M-1$, y $B$, que recibe los caudales combinados de los manantiales M-2, M-6, M-7 y M-8 (Fig. 1). El caudal de descarga del manantial M-3 se ha medido sólo en cuatro ocasiones debido a su descarga esporádica.

Se instaló un sensor de presión en la parte más profunda de la Laguna Tuyajto para obtener un registro continuo del nivel de la laguna. Para determinar la evaporación se utilizó un tanque de evaporación tipo A instalado a 4100 m s.n.m. al Norte del área de estudio.

\section{Resultados y discusión}

\section{Balance de agua de la Laguna Tuyajto}

La Laguna Tuyajto no tiene salidas superficiales. EI mayor aporte de agua proviene de los manantiales ubicados en sus bordes Norte y Este. La descarga total de los manantiales fluctúa entre 74 y $245 \mathrm{~L} / \mathrm{s}$, con una contribución total estimada para el año 2004 de $4.4 \mathrm{hm}^{3}$ (Tabla 1). La disminución sostenida del nivel de la laguna registrada entre diciembre de 2003 y enero de 2004 (Fig. 4) es consistente con los bajos caudales medidos en los manantiales a fines de enero del 2004 (90 L/s) y con una mayor evaporación registrada durante estos meses. El ligero aumento del nivel registrado en febrero y a mediados de marzo de 2004 es consistente con un pequeño aumento del caudal de los manantiales (Tabla 1) y con algunos eventos de precipitación registrados en febrero. Posteriormente se observa una tendencia ascensional desde finales de abril hasta septiembre del 2004, alcanzando hasta $62 \mathrm{~cm}$ de profundidad. Esto puede explicarse por la reducción de la tasa de evaporación en los meses del invierno austral (Tabla 2), en que las bajas temperaturas en algunos días de junio, julio y agosto congelan parcialmente la superficie de la Laguna y por el aumento de los caudales registrados en las estaciones $A$ y $B$, que se mantienen relativamente estables hasta septiembre, cuando empiezan a disminuir. El aumento del caudal puede explicarse por las lluvias ocurridas en febrero y la fusión de nieve que cayó durante los meses de julio y agosto. Finalmente, desde septiembre hasta fines de octubre del 2004 se observa una disminución del nivel de la laguna. Esta tendencia cambia a principios de 
Christian Herrera Lameli, et al., 2019. Investigaciones hidrogeológicas en la laguna... Boletín Geológico y Minero, 130 (4): $789-806$

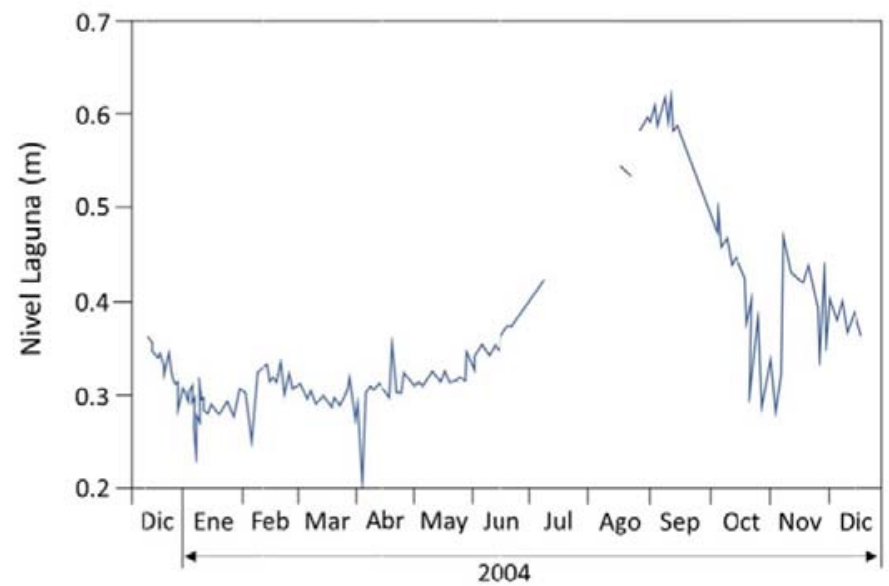

Figura 4. Fluctuación diaria del nivel de agua en la Laguna Tuyajto entre el 15 de diciembre de 2003 y el 19 de diciembre de 2004. Los niveles son relativos a una referencia altitudinal local.

Figure 4. Daily water level fluctuations in Laguna Tuyajto between December 15, 2003 and December 19, 2004. Levels are relative to a local altitudinal reference.

noviembre, cuando se registra un aumento significativo de nivel, consistente con un aumento significativo del caudal de los manantiales, especialmente el M1 (Estación A, Tabla 1).

\section{Flujo de agua subterránea}

En la Fig. 5 se observa el mapa piezométrico elaborado para las cuencas LagunaTuyajto, Pampa Las Tecas y Pampa Colorada. Los niveles indican la existencia de una conexión entre estas cuencas, con un flujo de agua con dirección y sentido general de Este a Oeste y cuyas descargas se producen en el sector oriental de la Laguna Tuyajto. Los bajos gradientes piezométricos en las cercanías de la laguna y en la zona central de las cuencas de Pampa Las Tecas y Pampa Colorada indican una transmisividad elevada asociada a los depósitos sedimentarios permeables, como gravas de antiguos abanicos aluviales, que es consistente con los resultados obtenidos de los ensayos de bombeo, los cuales indican transmisividades de hasta $1000 \mathrm{~m}^{2} / \mathrm{d}$ (WMC, 2006). El notable flujo puede explicar que no haya actualmente cuerpos salinos en las cuencas Pampa Las Tecas y Pampa Colorada y que los que pudiera haber habido ya fueron disueltos.

\section{Caracterización hidroquímica}

Las aguas subterráneas son del tipo clorurado sódico con un leve exceso de sulfato respecto a la relación

\begin{tabular}{|c|c|c|c|c|}
\hline Mes/Año & $\begin{array}{l}\text { Evaporación } \\
\text { en un tanque } \\
\text { tipo } \mathrm{A}(\mathrm{mm})\end{array}$ & $\begin{array}{c}\text { Evaporación } \\
\text { corregida } \\
(\mathrm{mm})\end{array}$ & $\begin{array}{c}\text { Área de } \\
\text { superficie } \\
\text { del lago } \\
\left(10^{4} \mathrm{~m}^{2}\right)\end{array}$ & $\begin{array}{c}\text { Volumen } \\
\text { de agua } \\
\text { evaporada } \\
\left(10^{3} \mathrm{~m}^{3}\right)\end{array}$ \\
\hline Ene/2004 & 217 & 134 & 112 & 150 \\
\hline Feb/2004 & 179 & 110 & 209 & 231 \\
\hline Mar/2004 & 208 & 128 & 134 & 172 \\
\hline Abr/2004 & 186 & 115 & 151 & 173 \\
\hline May/2004 & 164 & 101 & $200(*)$ & 203 \\
\hline Jun/2004 & 132 & 82 & $240(*)$ & 196 \\
\hline Jul/2004 & 136 & 84 & $250(*)$ & 210 \\
\hline Ago/2004 & 167 & 103 & $260(*)$ & 268 \\
\hline Sep/2004 & 204 & 126 & 272 & 343 \\
\hline Oct/2004 & 257 & 159 & 247 & 392 \\
\hline Nov/2004 & 291 & 180 & 245 & 440 \\
\hline Dic/2004 & 298 & 184 & $200(*)$ & 368 \\
\hline Total & 2439 & 1506 & & 3147 \\
\hline
\end{tabular}

Tabla 2. Evaporación media mensual de agua en la Laguna Tuyajto durante el año 2004. La evaporación corregida considera la salinidad del agua de la laguna. El área de la superficie de agua fue determinada a través de imágenes satelitales LANDSAT TM. (*) Valores aproximados del área derivados de mediciones del nivel. Table 2. Monthly average water evaporation in Laguna Tuyajto throughout 2004. The corrected evaporation is the result of considering lake water salinity. The surface area of water was determined through LANDSAT TM satellite images. (*) Approximate value of surface water area derived from measured water levels.

marina $\mathrm{rSO}_{4} / \mathrm{rCl}$ en algunas muestras. Las aguas de los manantiales ubicados en el sector Este de la LagunaTuyajto también son del tipo clorurado sódico y tienen la misma marca química que las aguas subterráneas en pozos de las cuencas Pampa Colorada y Pampa Las Tecas, mientras que los manantiales ubicados al Norte de la Laguna Tuyajto y en el Salar de Aguas Calientes son del tipo clorurado-sulfatado sódico. Las variaciones espaciales de la composición química de las muestras se observan en los diagramas de Stiff modificados (Fig. 6).

Los contenidos de $\mathrm{Na}$ y $\mathrm{Cl}$ están correlacionados positivamente para las aguas de los manantiales y de los pozos de Pampa Colorada y Pampa Las Tecas con salinidad baja y moderada. Sin embargo, existe un ligero exceso de cloruro respecto al sodio, especialmente para aguas con salinidad relativamente alta, que tiende a desaparecer en muestras con salinidad muy alta (Fig. 7). Los valores de la relación $\mathrm{rCl} / \mathrm{rBr}$ en los pozos de Pampa Las Tecas y Pampa Colorada varían de 2000 a 10000 , lo que es característico de la 


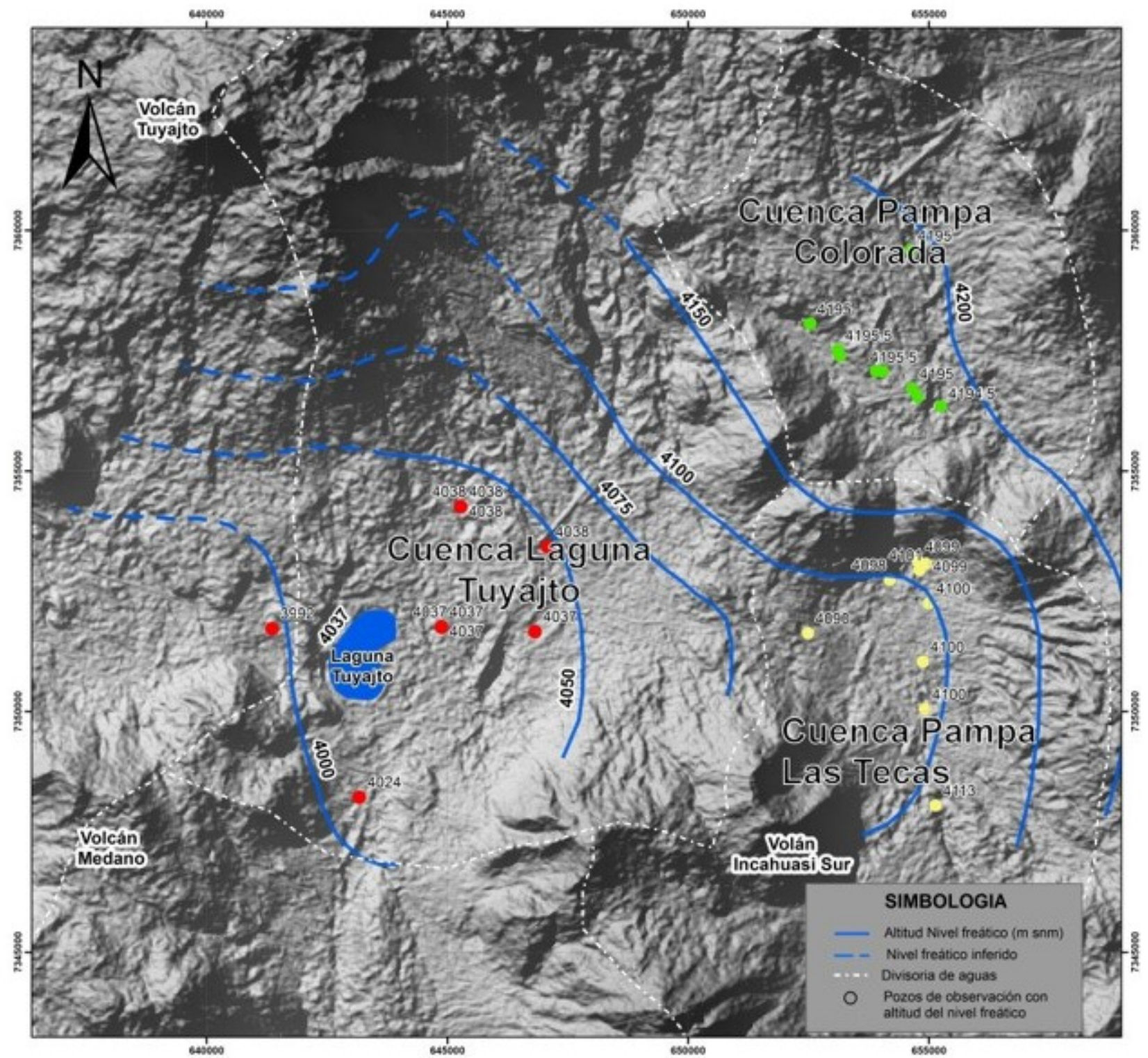

Figura 5. Mapa piezométrico del área. El flujo de aguas subterráneas es desde áreas de mayor recarga hacia zonas deprimidas (Este a Oeste).

Figure 5. Piezometric map of the area. The groundwater flows from areas of higher recharge to depressed areas (east to west).

disolución de halita (Fig. 8) (Custodio y Herrera, 2000; Alcalá y Custodio, 2008).

Los valores de la relación $\mathrm{rSO}_{4} / \mathrm{rCl}$ en muestras de lluvia varían de 0.26 a 0.28 , mientras que en los manantiales tienen un rango mayor, de 0.16 a 0.54 (Fig. 9). Las aguas subterráneas presentan una mayor variación de esta relación, de 0.01 a 0.45 . Las aguas con valores menores de la relación $\mathrm{rSO}_{4} / \mathrm{rCl}$ tienen una composición química cercana a la de las muestras de lluvia, mientras que algunos valores elevados de la relación que se encuentran en aguas diluidas pueden explicarse por meteorización de rocas volcánicas con sulfuros. Otros valores altos de $\mathrm{rSO}_{4} / \mathrm{rCl}$, que no se encuentran en agua diluida pueden ser producto de la difusión atmosférica de $\mathrm{SO}_{2}$ de oxidación de azufre nativo en una zona de alteración solfatárica que existe en la cima del volcán Tuyajto o de aportes endógenos. Considerando el carácter termal del manantial $\mathrm{M}-1$, localizado al Norte de la Laguna Tuyajto, no se puede descartar la disolución de $\mathrm{HCl}$ asociado al volcanismo reciente en la zona, como se ha reconocido en otras zonas volcánicas del 


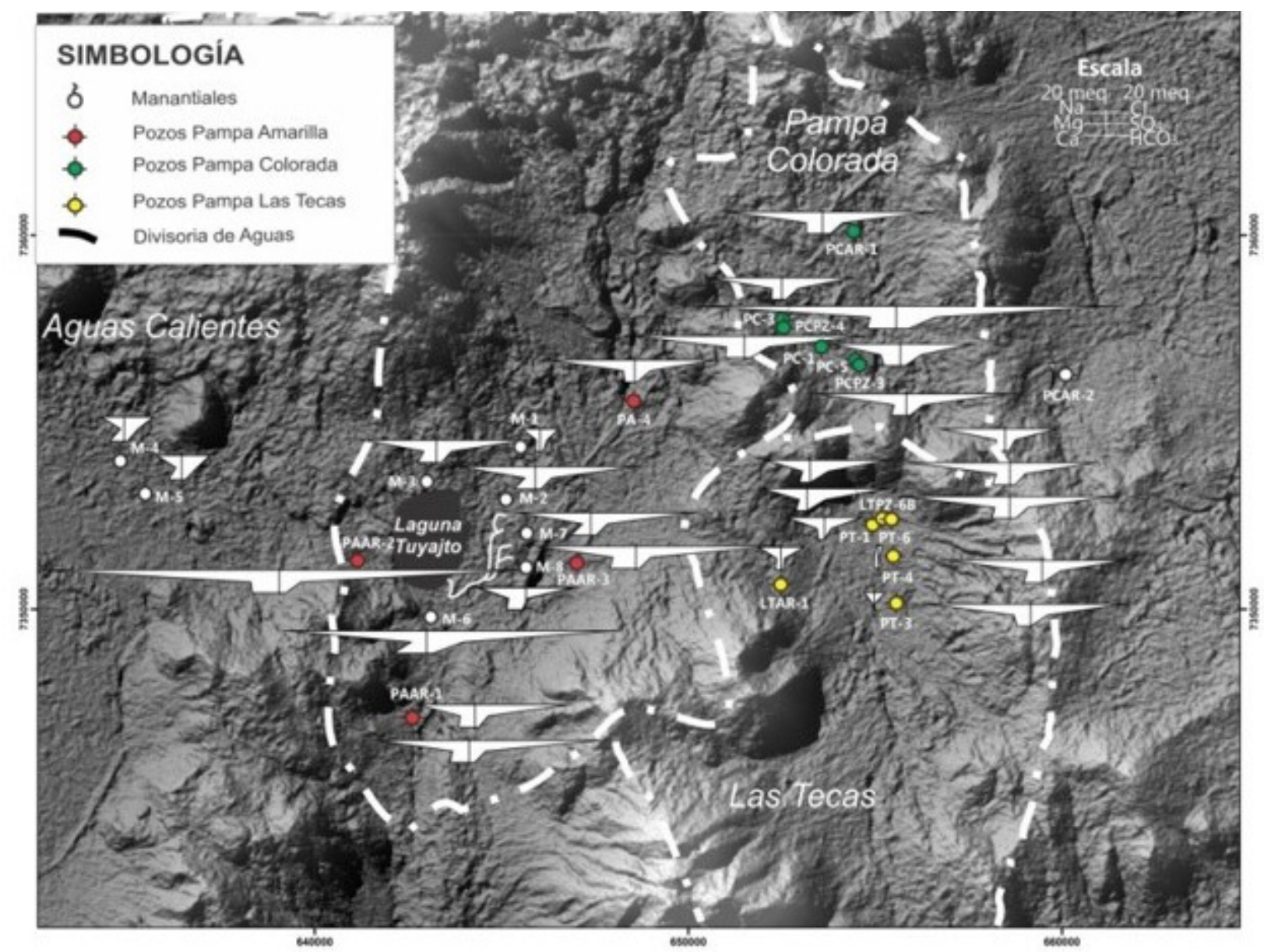

Figura 6. Diagramas de Stiff modificados de las aguas de manantiales ubicados en la Laguna Tuyajto y en el Salar de Aguas Calientes y de aguas de los pozos en Pampa Colorada, Pampa Las Tecas y Laguna Tuyajto. La divisoria de aguas indica el límite de las distintas cuencas hidrográficas superficiales.

Figure 6. Modified Stiff diagrams of waters of springs located in the Tuyajto Lagoon and the Salar (salt flat) de Aguas Calientes and wells in Pampa Colorada, Pampa Las Tecas and Laguna Tuyajto. The water divide indicates the divide between the surface watersheds.

Altiplano, como en los geiseres del Tatio (Cortecci et al., 2005).

$\mathrm{El} \mathrm{HCO}_{3}$ presenta valores relativamente pequeños en aguas de baja salinidad y asciende hasta aproximadamente 300 a $500 \mathrm{mg} / \mathrm{L}$ en aguas salinas y muy salinas. Las muestras de agua de pozos de Pampa Las Tecas y Pampa Colorada tienen un contenido elevado de $\mathrm{HCO}_{3}$ de aproximadamente $800 \mathrm{mg} / \mathrm{L}$, que podría explicarse por la incorporación de $\mathrm{CO}_{2}$ volcánico de desgasificación de las cámaras magmáticas y su posterior reacción con la roca para producir $\mathrm{HCO}_{3}$. Esto significaría que una parte del gas volcánico es transferida al agua subterránea de manera directa o por descarga lateral de agua subterránea desde los núcleos volcánicos, aun cuando la mayor parte de dicho gas sea emanada a la atmósfera. No existen determinaciones de ${ }^{13} \mathrm{C}$ para comprobar esta hipótesis.

\section{Isótopos estables de la molécula de agua}

Como resultado de la escasez de lluvias en el Norte de Chile, es difícil hacer una caracterización detallada del contenido isotópico de la precipitación. Para el transecto con grandes variaciones verticales en el que se ha muestreado la nieve, el $\delta^{18} \mathrm{O}$ varía entre 6.52 y $10.93 \%$, con un gradiente vertical isotópico de $\delta^{18} \mathrm{O}$ de $-2 \% / \mathrm{km}$, que es del mismo orden que los obtenidos en otras zonas de alta montaña, como los Alpes (Jódar et al., 2016a) o la cordillera Pirenaica (Jódar et al., 2016b). Las muestras de nieve son isotópicamente más ligeras que las de agua de Iluvia, cuyo $\delta^{18} \mathrm{O}$ varía entre -1.84 y $-10.31 \%$ (Fig. 10). En cualquier caso, los valores del contenido isotópico de las muestras de lluvia y nieve se encuentran entre la línea meteórica mundial y la línea meteórica local 


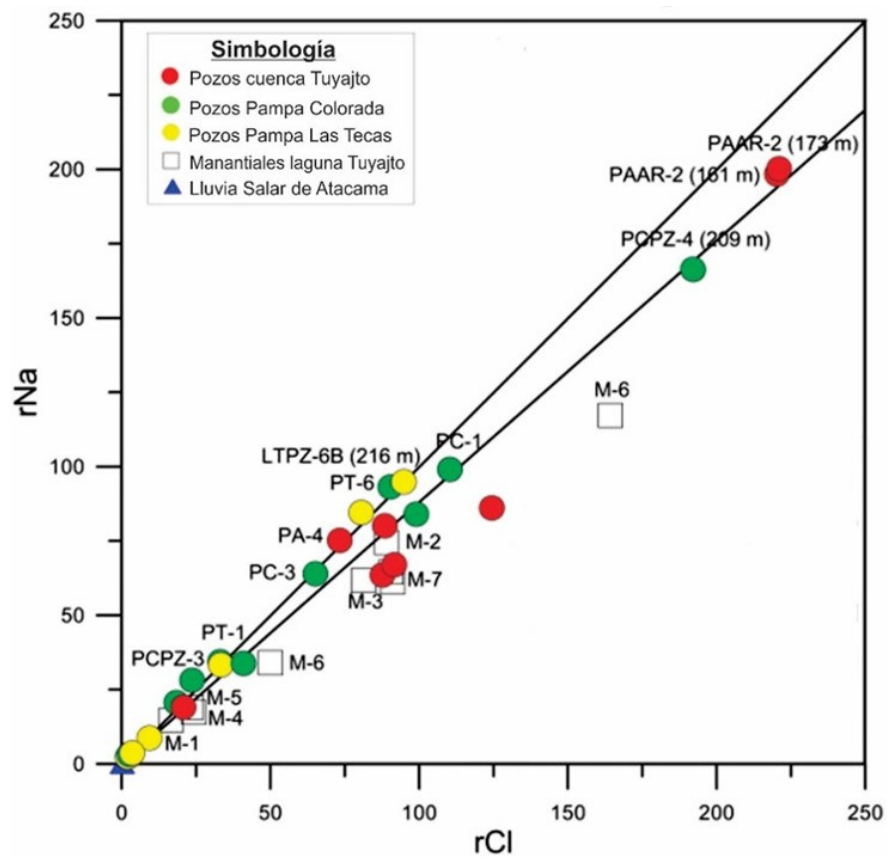

Figura 7. Gráfico de $r \mathrm{Na}$ en función de $\mathrm{rCl}(\mathrm{r}=\mathrm{meq} / \mathrm{L})$ de las aguas de manantial de la Laguna Tuyajto y de los pozos ubicados en las cuencas de Tuyajto, Pampa Colorada y Pampa Las Tecas. Algunas muestras presentan una relación $\mathrm{rNa} / \mathrm{rCl}$ menor que la relación 0,88 del agua oceánica media.

Figure 7. Plot of $r \mathrm{Na}$ vs. $r \mathrm{Cl}(r=m e q / L)$ of spring waters of Tuyajto lake and of the wells located in the Tuyajto, Pampa Colorada and Pampa Las Tecas basins. Some samples have a $\mathrm{rNa} / \mathrm{rCl}$ ratio less than 0.88 which characterize average oceanic water.

obtenida por Chaffaut (1998), de igual pendiente y exceso de deuterio $\left(\mathrm{d}=\delta^{2} \mathrm{H}-8 \cdot \delta^{18} \mathrm{O}\right)$ de $15.7 \%$.

El agua de los manantiales ubicados en la parte Norte de la Laguna Tuyajto y en el Salar de Aguas Calientes 3 (M-1, M-3, M-4 y M-5) tiene una composición isotópica cercana al agua meteórica, aunque muy ligera, lo que indica que el agua de recarga de estos manantiales procede mayoritariamente de la fusión de nieve en las laderas del volcán Tuyajto a cotas superiores a $4900 \mathrm{~m}$ s.n.m. El contenido isotópico del agua de los manantiales M-2, M-7 y M-6 apunta la existencia de un proceso de evaporación. EI manantial M-6, teniendo en cuenta la piezometría (Fig. 5), podría resultar de un proceso de mezcla entre el agua de recarga y el agua de la laguna. Esto implicaría que la laguna del Tuyajto tendría un funcionamiento del tipo de tránsito (a través o flowthrough, Sophocleous, 2009), esto es, el nivel de la laguna dependería de la descarga de los manantiales a la vez que recarga el acuífero subyacente, con flujo hacia otros lugares.

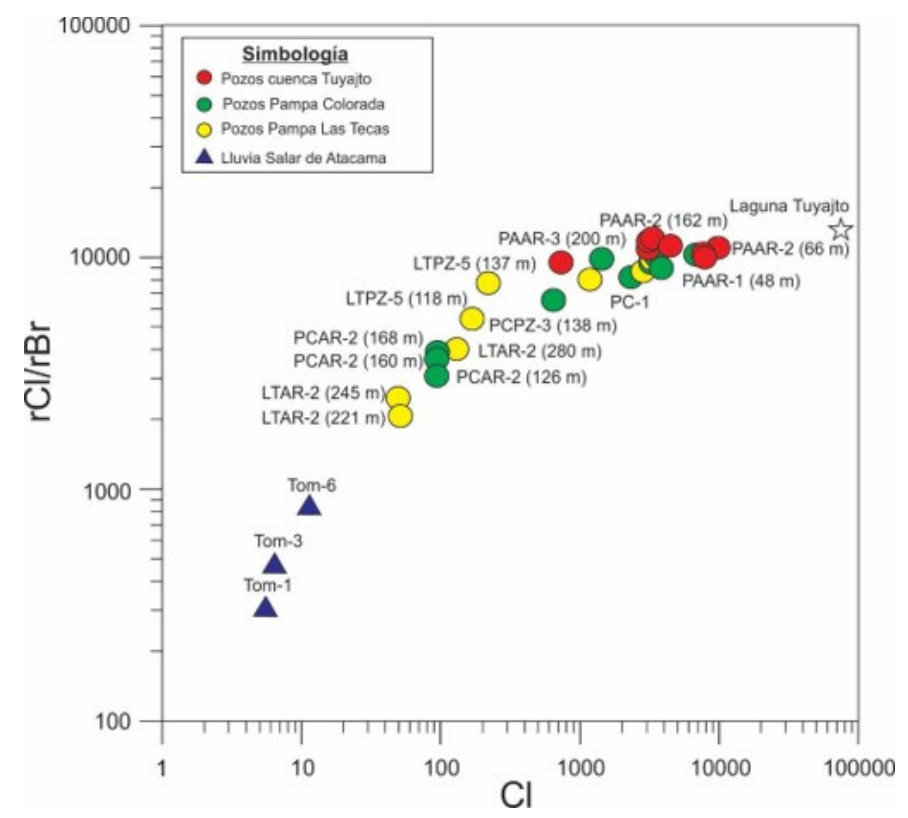

Figura 8. Gráfico de la relación $\mathrm{rCl} / \mathrm{rBr}$ en función del $\mathrm{Cl}$. Muestra un aumento general de $\mathrm{rCl} / \mathrm{rBr}$ al aumentar la salinidad. Se incluyen datos de lluvia del flanco Oeste del Salar de Atacama. $r=m e q / L$. Los valores en paréntesis son la profundidad a la cual se tomó la muestra de agua.

Figure 8. Plot of the $\mathrm{rCl} / \mathrm{rBr}$ ratio versus $\mathrm{Cl}$. It shows a general increase of $\mathrm{rCl} / \mathrm{rBr}$ as salinity augments. Rainfall data on the western flank of the Atacama salt flat are included. $r=m e q / L$. Values in parentheses show the depth at which the water sample was taken.

Las muestras de agua subterránea de Pampa Colorada, Pampa Las Tecas y el manantial M-8 tienen composiciones isotópicas enriquecidas en $\delta^{18} \mathrm{O}$ con respecto al agua meteórica. Una explicación es que el agua meteórica original que recarga el sistema acuífero haya sido afectada por intercambio isotópico profundo de alta temperatura con la roca, lo que es plausible teniendo en cuenta el entorno volcánico del acuífero y la existencia de aguas termales en las cercanías de la Laguna Tuyajto. El valor más negativo de $\delta^{2} \mathrm{H}$ de algunas muestras obedecería a la composición isotópica más ligera del agua meteórica original. EI contenido isotópico de $\delta^{18} \mathrm{O}$ en muestras de nieve en función de la altitud indican una recarga preferente para las aguas de manantiales entre 4100 y 4700 m s.n.m.

Considerando el $\delta^{2} \mathrm{H}$ se obtienen altitudes de recarga preferente entre 4100 y 4600 m s.n.m. Estas altitudes hay que relativizarlas en función del efecto de ladera, aunque en este caso el efecto nival se concentra a las alturas superiores. 


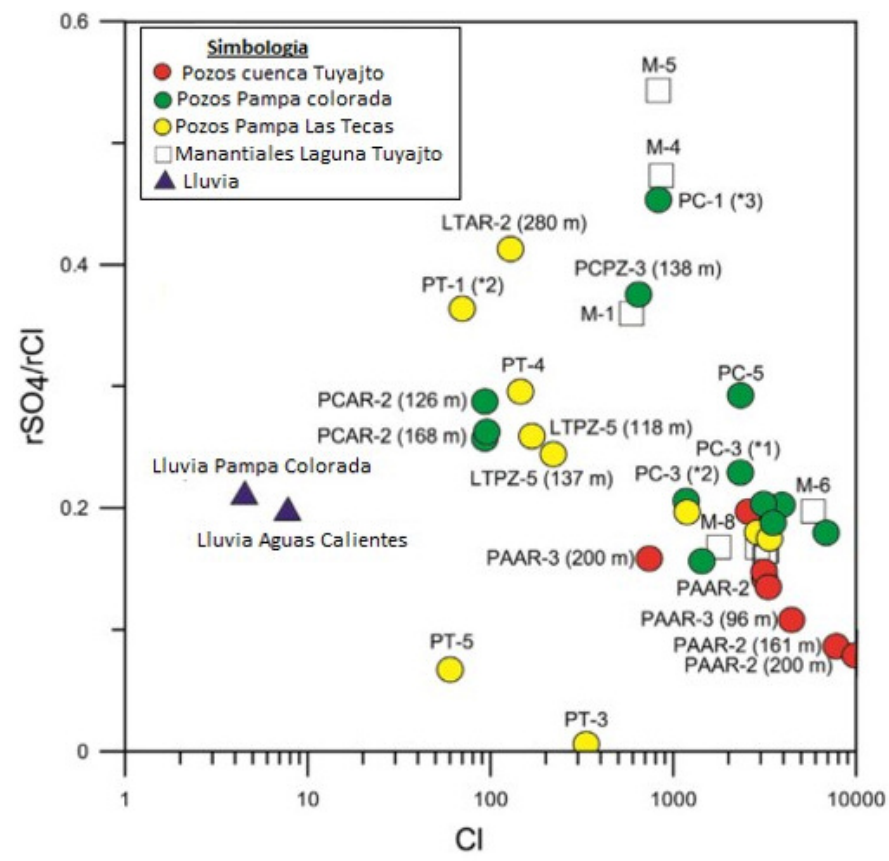

Figura 9. Gráfico de la relación $\mathrm{rSO}_{4} / \mathrm{rCl}(\mathrm{r}=\mathrm{meq} / \mathrm{L})$ en función del $\mathrm{Cl}$ ( $\mathrm{mg} / \mathrm{L}$ ) de las aguas de los manantiales de la Laguna Tuyajto y de los pozos ubicados en las cuencas de Tuyajto, Pampa Colorada y Pampa Las Tecas.

Figure 9. Plot of the $\mathrm{rSO}_{4} / \mathrm{rCl}$ ratio $(r=m e q / L)$ versus $\mathrm{Cl}(\mathrm{mg} / \mathrm{L})$ of spring waters of Tuyajto lake and of the wells located in the Tuyajto, Pampa Colorada and Pampa Las Tecas basins.

\section{Caracterización de la recarga}

El caudal de los manantiales muestra que la recarga es importante a pesar de la aridez imperante en la zona. Para una superficie de recarga entre 400 a 600 $\mathrm{km}^{2}$ y una descarga media en manantiales de 3 $\mathrm{hm}^{3} / a n ̃ o$, la recarga media puede estimarse de forma aproximada entre 5 y $7.5 \mathrm{~mm} / \mathrm{año}$, lo que corresponde a un porcentaje de la precipitación media anual entre el $2 \%$ y el $4 \%$. Este valor puede ser mucho más en las zonas de mayor altitud y bastante menor en las depresiones (Custodio, 2010). La precipitación promedio en la zona de estudio varía entre 150 y 200 $\mathrm{mm} / \mathrm{año}$. Teniendo en cuenta que la concentración promedio de ion cloruro en las muestras de agua de lluvia es de $5 \mathrm{mg} / \mathrm{L}$ (Risacher et al., 1999), se obtiene una tasa de deposición de cloruros entre 0.75 y $1 \mathrm{~g} \mathrm{~m}$ ${ }^{2}$ año ${ }^{-1}$. Este valor parece alto si se considera que las precipitaciones tienen un origen Atlántico, ya que las masas de aire húmedo han de cruzar toda la cuenca del Amazonas antes de producir precipitación en la zona de estudio. Sin embargo, este valor es razonable si se considera que 1) la recarga puede ser en parte debida a la fusión de nieve que precipita durante el invierno austral (Ammann et al., 2001) debido al paso de los sistemas frontales fríos provenientes del

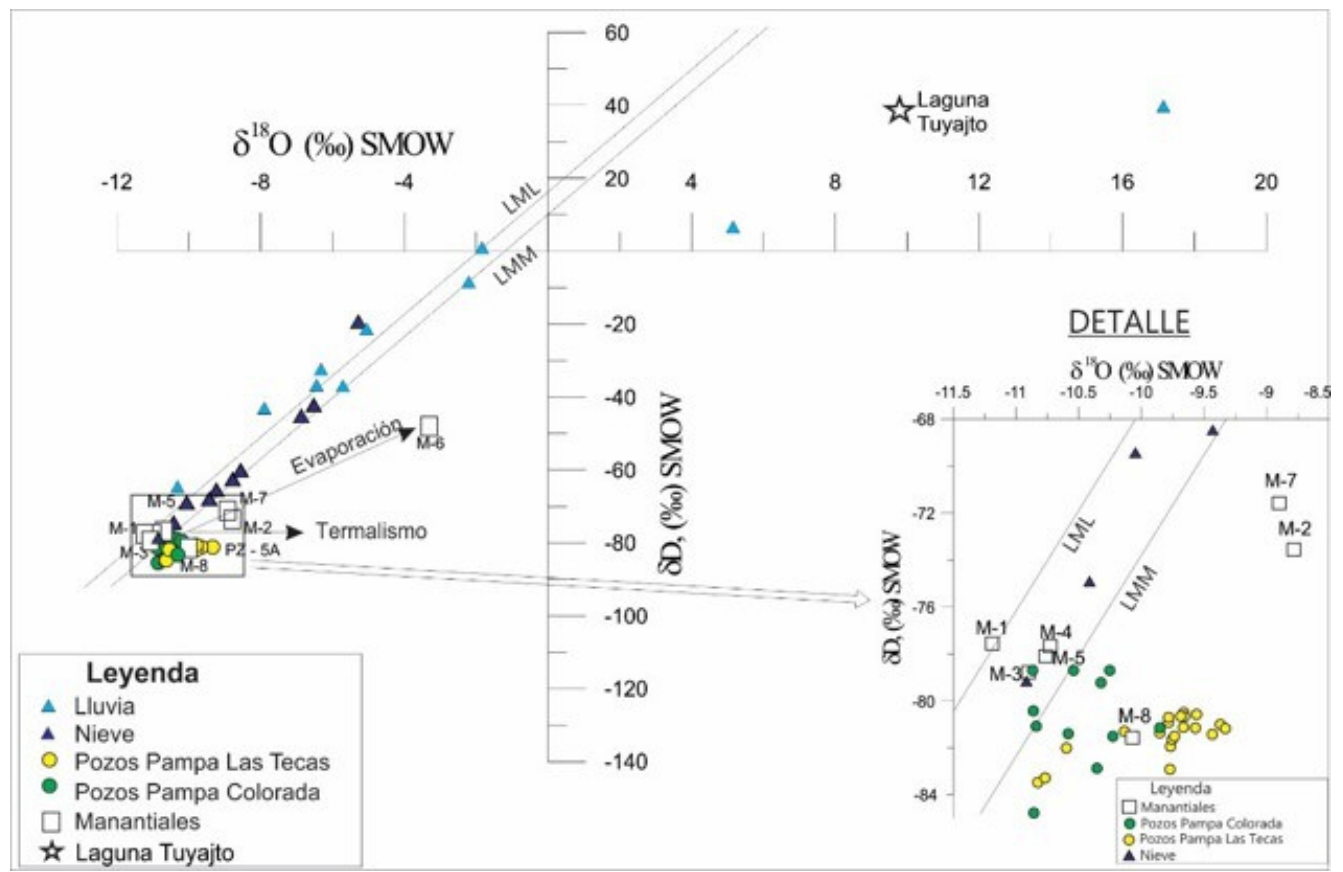

Figura 10. Composición isotópica de $\delta^{18} \mathrm{O}$ y $\delta^{2} \mathrm{H}(\delta \mathrm{D})$ de aguas de manantiales, precipitaciones en el área y de aguas de pozos. LMM: Línea meteórica mundial. LML: Línea meteórica local, paralela con un exceso de deuterio de 15,7\% SMOW.

Figure 10. Isotopic composition of $\delta^{18} \mathrm{O}$ and $\delta^{2} \mathrm{H}(\delta \mathrm{D})$ of spring waters, precipitation in the area and well water. LMM: world meteoric line. LML: Local meteoric line, parallel with a deuterium excess of $15.7 \%$ SMOW. 
Pacífico (Vuille y Ammann, 1997) y 2) a que la emanación de gases volcánicos del entorno puede aumentar localmente el contenido atmosférico en $\mathrm{Cl}$, además de en S.

La composición isotópica de $\delta^{18} \mathrm{O}$ y $\delta^{2} \mathrm{H}$ en las muestras de precipitación y aguas subterráneas del área muestra cómo la fusión de nieve que se deposita en las laderas del volcán Tuyajto juega un rol importante en la recarga del acuífero. Esta recarga se suma a la que produce la precipitación a lo largo de la pendiente. Como resultado, y favorecido por el efecto ladera (Custodio y Jódar, 2016), el agua que descarga en los manantiales a cotas bajas es menos salina e isotópicamente más ligera que el agua de la recarga local.

Las determinaciones de tritio reportadas por WMC (2006) para las aguas muestreadas en los pozos de las cuencas de Pampa Colorada, Pampa Las Tecas y Tuyajto indican que el periodo de renovación corres- pondiente podría ser de más de tres décadas, ya que la mayoría de las muestras tienen concentraciones de tritio por debajo de 0.8 UT ( 1 UT, unidad de tritio = $\left.10^{-18}{ }^{3} \mathrm{H} / 1 \mathrm{H}\right)$ que es el umbral de medición del contenido en tritio en el agua con las técnicas analíticas usadas en el laboratorio. La Fig. 11 presenta un esquema del modelo conceptual desde el volcán Tuyajto hasta la laguna.

\section{Temperatura del agua subterránea}

La temperatura del agua en los pozos de la cuenca Pampa Colorada y de los manantiales del borde Norte de la Laguna Tuyajto es superior a $20^{\circ} \mathrm{C}$ y en algunos casos es cercana a $40^{\circ} \mathrm{C}$. Estos valores elevados se encuentran cerca del volcán Tuyajto (especialmente los manantiales $\mathrm{M}-1$ y $\mathrm{M}-3$ ) y posiblemente son resultado de la reciente actividad magmática. Estas tem-

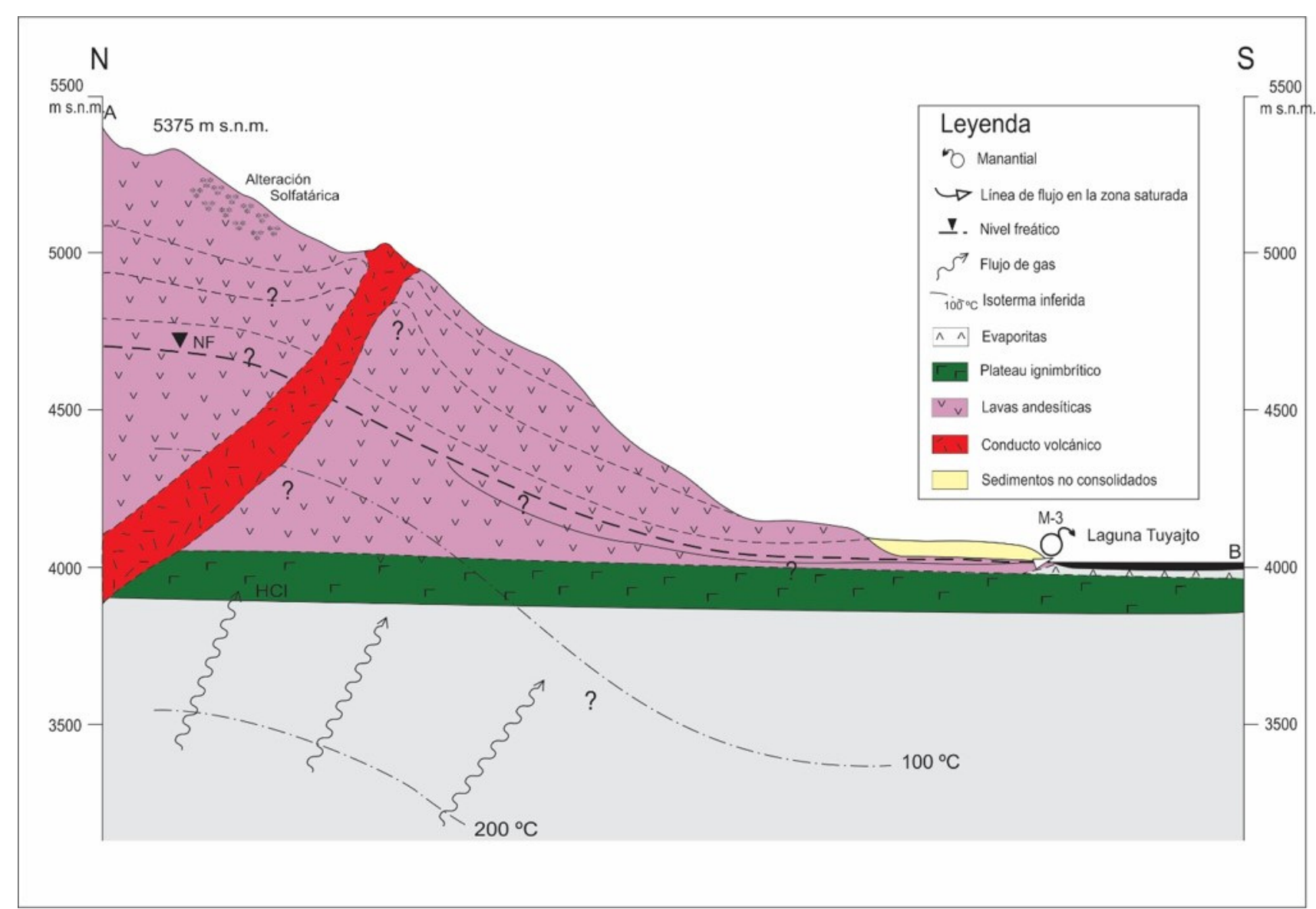

Figura 11. Esquema del modelo conceptual de la cuenca Tuyajto, desde el volcán Tuyajto hasta la laguna, mostrando el rol de la planicie ignimbrítica de baja permeabilidad en el flujo de las aguas subterráneas.

Figure 11. Diagram of the conceptual model of the Tuyajto basin, from the Tuyajto volcano to the lagoon, showing the role of the low permeability ignimbrite plateau in the flow of groundwater. 
peraturas son mayores a las medidas en los pozos de la cuenca Pampa Las Tecas, que se encuentran dentro del rango de 10 y $20^{\circ} \mathrm{C}$. Adicionalmente, las aguas de manantiales que descargan en el borde oriental de la Laguna Tuyajto no muestran efectos hidrotermales claros, exceptuando el manantial M-8, aunque muestran valores de $\delta^{18} \mathrm{O}$ con tendencia a contenidos más pesados (Fig. 10). Lo anterior puede indicar un intercambio isotópico de interacción agua-roca que está ocurriendo lejos del área de descarga y enfriándose a lo largo de su trayectoria. Se puede obtener una estimación aproximada de la temperatura original mediante geotermómetros. Para la interpretación se supone lo siguiente: a) equilibrio químico e isotópico a alta temperatura con los minerales existentes, b) rápido ascenso de agua a la superficie, de modo que no hay tiempo para cambiar la composición química e isotópica de las aguas profundas de manera significativa y c) no hay mezcla con otras aguas ni evaporación (Custodio y Llamas, 1983). Las condiciones antes descritas deben comprobarse en cada escenario. Considerando lo anterior, el geotermómetro de cuarzo no pudo ser aplicado por: 1) falta de equilibrio con cuarzo o cualquier forma de sílice ya que las rocas dominantes en el área son andesitas y basaltos y 2) alta concentración de sales, como la halita, cuya disolución en agua dificulta el uso de geotermómetros basados en cationes (Giggenbach, 1988).

\section{Relación entre la salmuera de la Laguna Tuyajto y el sistema de aguas subterráneas}

La salmuera de la Laguna Tuyajto se encuentra cerca de la saturación con respecto a la halita. En acuíferos con un espesor importante, la alta concentración en sales disueltas en la capa superior en que se produce la evaporación podría dar lugar a flujos de convección en la laguna, en cuyo caso provocarían un flujo descendente de la salmuera hacia aguas con menor concentración y menor densidad, desplazándolas, como consecuencia de la diferencia entre las densidades (Fan et al., 1997). Este tipo de flujos son comunes en muchas regiones salinas (Duffy y Al-Hassan, 1988) y está caracterizado en el Salar de Atacama, al Oeste, que es el fondo de cuenca terminal de todo el sistema, Pero la escasa profundidad del agua y lo somero del nivel ignimbrítico de baja permeabilidad que conforma el fondo de la Laguna Tuyajto dificulta la generación de dichos flujos convectivos de la salmuera. Sin embargo, estos flujos convectivos se pueden producir en el acuífero a escala mayor. que comprende las cuencas altiplánicas.

Puede existir una transferencia de agua desde la
Laguna Tuyajto hacia el Salar de Aguas Calientes 3 (Herrera et al., 2016), debido a la diferencia topográfica entre ambas cuencas y a la alta concentración de cloruro sódico que se observa en el pozo PAAR-2 (Fig. 6). Sin embargo, la alta acumulación de sales en la Laguna Tuyajto, así como también la naturaleza del material volcánico que limita el Oeste de la cuenca, parece indicar que esta transferencia es pequeña. Lo anterior permite deducir un comportamiento tipo tránsito de la laguna respecto el flujo regional del acuífero.

\section{Conclusiones}

La composición química de las aguas de manantiales de la Laguna Tuyajto y de los pozos de las cuencas Pampa Colorada y Pampa Las Tecas estaría controlada por la disolución de minerales evaporíticos previos enterrados bajo depósitos volcánicos y derivados más recientes, por la evaporación de aguas meteóricas y en parte por la interacción agua-roca a alta temperatura. El exceso de cloruro sobre el ion sodio respecto a la relación marina podría ser debido a cambios catiónicos, pero también cabe considerar que sea debido a la incorporación de $\mathrm{HCl}$ de desgasificación magmática, aunque esto aún se está por estudiar.

El contenido isotópico de las muestras de agua correspondientes a los manantiales ubicados en el Noreste y Norte de la laguna de Tuyajto indica que el agua de recarga procede básicamente de la fusión de la nieve acumulada a altas cotas en la ladera Sur del volcán Tuyajto. Esta nieve procede en parte de los eventos de precipitación generados por los frentes fríos que parten desde el Pacífico y barren la zona de estudio en invierno. Adicionalmente y en menor medida, el agua de lluvia procedente de eventos intensos de precipitación que típicamente acontecen en verano también puede producir recarga a lo largo de la pendiente existente desde las zonas altas de la cuenca hasta el fondo de esta, donde se encuentra la laguna.

El agua muestreada en los manantiales ubicados en el lado Este de la LagunaTuyajto es el resultado de un proceso de mezcla entre el agua de recarga producida por la precipitación y aguas subterráneas procedentes de la transferencia lateral de las cuencas hidrológicas vecinas de Pampa Las Tecas y Pampa Colorada.

La laguna de Tuyajto muestra localmente un funcionamiento tipo transito respecto el flujo regional del acuífero. Su existencia y dinámica depende esencialmente de las aportaciones de los manantiales y de 
la transferencia de agua desde las cuencas vecinas de Pampa Las Tecas y Pampa Colorada. La conservación de los valores ecológicos y de avifauna de la laguna Tuyajto estaría por lo tanto muy ligada a los cambios territoriales del entorno y en especial la posible explotación de agua subterránea.

\section{Agradecimientos}

Esta investigación se ha llevado a cabo en el marco del Proyecto Anillo ACT1203 de la CONICYT de Chile. La investigación también fue financiada por una beca de doctorado CONICYT-PCHA / Doctorado Nacional / 2015-21150951 de J.U. y una beca de doctorado CONICYT-PCHA / Doctorado Nacional / 2016-21160152 de C.G.

\section{Referencias}

Alcalá, F.J, Custodio, E. (2008). Using the $\mathrm{Cl} / \mathrm{Br}$ ratio as a tracer to identify the origin of salinity in aquifers in Spain and Portugal. Journal of Hydrology, 359 (1-2): 189207.

Ammann, C., Jenny, B., Kammer, K., Messerli, B. (2001). Late Quaternary glacier response to humidity changes in the arid Andes of Chile (18-29 ${ }^{\circ}$ S). Paleogeography, Paleoclimatology, Paleoecology, 172(3-4): 313-326.

Backer, M.C. (1981). The nature and distribution of upper Cenozoic ignimbrite centres in the Central Andes. Journal of Volcanology and Geothermal Research, 1981; 11(2-4): 293-315.

Chaffaut I. (1998). Precipitations d'altitude, eaux souterraines et changements climatiques de I'Altiplano NordChilien. Thèse de Doctorat Université Paris XI Orsay: 1274.

Chong, G. (1988). The Cenozoic saline deposits of the Chilean Andes between 1800 and 2700 south latitude. In The Southern Central Andes, Springer Berlin Heidelberg: 137-151.

Cortecci, G., Boschetti, T., Mussi, M., Herrera C., Mucchino, C., Barbieri, M. (2005). New chemical and original isotopic data on waters from El Tatio geothermal field, northern Chile. Geochemical Journal, 39: 547-571.

Custodio, E., Llamas, M.R. (1983). Hidrología subterránea. Editorial Omega, Barcelona: 1-2350.

Custodio, E., Herrera, C. (2000). Utilización de la relación $\mathrm{Cl} / \mathrm{Br}$ como trazador hidrogeoquímico en hidrología subterránea. Boletín Geológico y Minero, 111(4): 49-67.

Custodio, E. (2010). Estimation of aquifer recharge by means of atmospheric chloride deposition balance in the soil. Constributions to Science, 2010, 6(1): 81-97.

Custodio, E., Jódar, J. (2016). Simple solutions for steadystate diffuse recharge evaluation in sloping homogeneous unconfined aquifers by means of atmospheric tracers. Journal of Hydrology, 540: 287-305.

DGA (Dirección General de Aguas) (2009). Información
Oficial Hidrometeorológica y de Calidad de Aguas (on line). Ministerio de Obras Públicas. Santiago, Chile. http://snia.dga.cl/BNAConsultas.

Duffy, C., Al-Hassan, S. (1988). Groundwater circulation in a closed desert basin: Topographic scaling and climatic forcing. Water Resources Research, 24: 1675-1688.

Fan, Y., Duffy, C. J., Oliver, D.S. (1997). Density-driven groundwater flow in closed desert basins: field investigations and numerical experiments. Journal of Hydrology, 196(1): 139-184.

Geyh, M., Grosjean, M., Nuñez, L., Schotterer, I. (1999). Radiocarbon reservoir effect and the timing of the LateGlacial/Early Holocene humid phase in the Atacama Desert (Northern Chile). Quaternary Research, 52: 143153.

Giggenbach, W. (1988). Geothermal solute equilibria. Derivation of Na-K-Mg-Ca geoindicators.

Geochimica et Cosmochimica Acta, 52: 2749-2765.

Herrera, C., Custodio, E., Chong, G., Lambán, L.J., Wilke, H., Riquelme, R., Jódar, J., Urrutia, J., Urqueta, H., Gamboa, C., Sarmiento, A., Lictevout, E. (2016). Groundwater flow in a closed basin with a saline shallow lake in a volcanic area: Iaguna Tuyajto, northern chilean altiplano of the Andes. Science of The Total Environment, 541, 303-318.

Jódar, J., Custodio, E., Liotta M., Lambán, J.L., MartosRosillo, S., Sapriza, G., Rigo, T., Herrera, C. (2016a). Correlation of the seasonal isotopic amplitude of precipitation with annual evaporation and altitude in alpine regions. Science of The Total Environment, 550: 27-37.

Jódar, J., Custodio, E., Lambán, J.L., Martos-Rosillo, S., Herrera, C., Sapriza, G. (2016b). Vertical variation in the amplitude of the seasonal isotopic content of rainfall as a tool to jointly estimate the groundwater recharge zone and transit tid ${ }^{18} \mathrm{Omes}$ in the Ordesa and Monte Perdido National Park aquifer system, north-eastern S pain. Science of The Total Environment, 573: 505-517.

Lindsaya, J.M., de Silva, S., Trumbulla, R., Emmermanna, R., Wemmerc, K. (2001). La Pacana caldera, N. Chile: a reevaluation of the stratigraphy and volcanology of one of the world's largest resurgent calderas. Journal of Volcanology and Geothermal Research, 106: 145-173.

Mardones, L. (1977). Geología e hidrogeología de los salares de Ascotán y Carcote, II Región, Chile. Universidad Católica del Norte. Memoria de Título: 1-234.

Montgomery, E., Rosko, M., Castro, S., Keller, B., Bevacqua, P. (2003). Interbasin underflow between closed Altiplano basins in Chile. Groundwater, 41: 523-531.

Ramírez, C., Gardeweg, M. (1982). Hoja Toconao, Región de Antofagasta. Servicio Nacional de Geología y Minería, Carta Geológica de Chile, No. 54: 1-122.

Risacher, F., Alonso, H., Salazar, C. (1999). Geoquímica de aguas en cuencas cerradas: I, II y III Regiones - Chile, Dirección General de Aguas: 1-461.

Risacher, F., Alonso, H., Salazar, C. (2003). The origin of brines and salts in Chilean salars: a hydrochemical review. Earth-Science Reviews, 63(3-4): 249-293.

Sophocleous, M. (2009). Relationships between groundwater and surface water in the Prairie Wetlands of North America. IAH-Spanish Chapter. October 2009. Zaragoza. Stern, C., Moreno, H., López-Escobar, L., Clavero, J., Lara, 
L., Naranjo, J., Parada, M., Skewes, A. (2007). Chilean volcanoes. In: Moreno, T., Gibbons, W. (ed.), Geology of Chile, Geological Society of London: 289-308.

Vuille, M., Ammann. C. (1997). Regional snowfall patterns in the high, arid Andes. Clim. Change., 36: 413-423.
WMC (Water Management Consultants) (2006). Evaluación de impactos hidrológicos producto de la extracción de agua Subterránea - Proyecto Pampa Colorada. Environmental Impact Study for Pampa Colorada (EIA Pampa Colorada) for Minera Escondida Ltda.

Recibido: julio 2018

Revisado: septiembre 2018

Aceptado: febrero 2019

Publicado: diciembre 2019 
